\title{
The Prospect of Expert System-Based Cognitive Support as a By-Product of Image Acquisition and Reporting
}

\author{
Pradeep G. Mutalik, Gregory G. Weltin, Paul R. Fisher, and Henry A. Swett
}

\begin{abstract}
In order for computer-based decision-support tools to find routine use in the everyday practice of clinical radiology, further development of user interface and knowledge content are required. In an ideal interface, the interaction between the radiologist and the computer would be minimized and painlessly integrated into existing work patterns. In this article, we explore some of the ways that pre-existing computer interactions in the processes of image acquisition and reporting can be used to feed case information into an expert system and thereby allow users to acquire advice from it in an automatic fashion. We describe interface models that we have developed in the domains of mammography and obstetric ultrasound, and discuss interface and content-related questions that have arisen from informal evaluations of these systems. In particular, the need for clinical outcome-relevant decision support and training level-appropriate decision support are discussed in detail.
\end{abstract}

Copyright $\odot 1991$ by W.B. Saunders Company

KEY WORDS: expert systems, man-machine interface, decision support, image acquisition, image reporting. mammography, obstetric ultrasound.

$\mathrm{C}$ OMPUTER-BASED decision-support tools in radiology date back to a system developed by Lodwick in 1963 that calculated the probability of bone tumor diagnoses based on observed findings. ${ }^{1}$ In more recent times, prototype expert systems have appeared that use artificial intelligence techniques to provide support for various cognitive tasks in radiology, such as test ordering ${ }^{2,3}$ and differential diagnosis..$^{4-9}$ More recently, newer methods such as artificial neural networks have been used in computer-based decision-support systems in radiology. ${ }^{10-12}$ In general these systems are meant to be used off-line, that is, case information must be entered into them by the radiologist whenever he encounters a case where he or she feels that the system can be useful. This requires interaction with the system solely for this purpose and therefore represents an interruption in the radiologist's work flow. This important factor may inhibit the routine clinical use of such systems.

Nevertheless, we see computer systems everywhere in radiology today, perhaps more so than in any other branch of medicine. Computers are routinely used to acquire radiological images (eg, computed tomography, magnetic resonance, ultrasound, etc), in radiology information systems, and more recently are beginning to be used in image archiving [picture archiving communication system (PACS) ] and in resultreporting (computerized voice recognition). We have sought to make use of these pre-existent computer interactions and build computerbased expert systems that can give a radiologist intelligent decision support without intruding on his or her normal film-reading activity and without requiring specific and lengthy computer interaction. Our prototype systems use the computer interactions already being performed for image acquisition and reporting to acquire case knowledge about the film being interpreted. This knowledge can then be used by a computer system working in the background to generate intelligent diagnostic advice. The radiologist thus gets the benefit of automatic expert system consultation without having to do anything different from what he or she normally does during the film-reading process.

In this article, we describe these systems as representatives of the efforts being made by ourselves and others to overcome the manmachine interface problem. We also address some of the deficiencies that remain, despite our best efforts, in expert system design and content. Many of the hurdles are technological (eg, speech recognition) and as designers of expert systems, we have no control over them. However, we argue that there are several issues relating to expert system design and the manmachine interface that have not been addressed adequately in conventional medical expert systems, such that even if we had the requisite technologic tools at our disposal today, it would still not be possible to construct a useful intelli-

From the Department of Diagnostic Radiology, Yale University School of Medicine, New Haven, CT.

Address reprint requests to Dr Pradeep G. Mutalik, Diagnostic Radiology BML 332, Yale University School of Medicine, 333 Cedar St, New Haven, CT 06511.

Copyright $(1991$ by W.B. Saunders Company

0897-1889/91/0404-0006\$03.00/0 
gent radiologic workstation. Hence, we endeavor to list some issues that must be addressed by expert system designers to make full use of future technologies. The interface issues are not the only hurdles to be overcome-the decision-support systems themselves must become more robust and cater to operators at different levels of expertise. This analysis is based on our experiences in designing and informally evaluating the IMAGE/ICON systems (prototype expert systems that provide both text as well as image output as an aid to radiologic differential diagnosis) in three domains within radiology: lymphoproliferative disorders in chest radiography, ${ }^{4.5}$ mammography, ${ }^{13}$ and, recently, obstetric ultrasound.

\section{THE MAN-MACHINE INTERFACE}

An ideal man-machine interface would allow the computer to acquire enough case information in some manner not involving a lengthy question-and-answer interaction with the radiologist for this specific purpose. This is especially true of systems designed to help with fairly common problems; systems dealing with unusual, clearly delineated problems like bone tumor diagnosis might be acceptable as off-line aids. One way for on-line systems to acquire case information is by extracting such information directly from the film. Another way is for the computer to acquire such information during the performance of some other essential computer-based function, such as image acquisition, information-system interaction, or during voice reporting.

\section{Computer Vision}

Extracting radiologically relevant material directly from the digitized image would provide an ideal input to a computer-based expert system. However, this is a difficult problem and the most sophisticated algorithms running on the fastest computers still cannot compare in visual pattern recognition with the human brain-even one that is only 3 years old. It is safe to say that of all the technologies we discuss here, this one will take longest to mature fully, and fully computerized film reading is not likely in the foreseeable future.
Nevertheless, there have been isolated successes in computer recognition of specific pathologies such as breast masses ${ }^{14}$ and calcifications ${ }^{15}$ in the domain of mammography, and pulmonary nodules ${ }^{16}$ and interstitial lung disease ${ }^{17}$ in chest radiology, among others. With the advent of PACS technology and direct digital image acquisition, initial screening of digital images could well be performed by computer, with the purpose of alerting the radiologist to potential lesions. Some preliminary radiologic information may be obtained by the expert system in this way, but for more detailed information it will have to depend on superior human visual pattern recognition skills.

\section{Case Input During Image Acquisition}

Most radiologic imaging techniques are fairly operator independent; standard technical parameters will produce an image that satisfactorily records any findings present. On the other hand, ultrasound examinations depend on the skill and knowledge of the examiner. A sonographer doing an obstetric ultrasound examination must have a thorough knowledge of anatomy and some knowledge of pathology in order to obtain a complete examination. In this modality a sonographer typically acquires several images of various parts of the fetus and uterus along different axes. We have developed a prototype system that not only provides guidance intended to help the sonographer recognize abnormalities and obtain a complete examination, but also facilitates documentation of observed findings. This last feature can allow higher level expert system advice (and reference images) to be presented to the radiologist together with the images.

The working screen is divided into four windows: the real-time display, the uterine survey, the view agenda, and the measurements window (Fig 1). The real-time window in our model is a slaved real-time image from the ultrasound machine with buttons serving as operator controls. This would enable the operator to carry out the entire examination from workstation screen alone. The view agenda and measurements windows are meant to be intelligent checklists. They remind the technologist of all the views and measurements required for a 
Fig 1. OB/ONE working screen showing (clockwise from upper left) the real-time window, the uterine survey, measurements, and view agenda windows. The icons for the obtained views are checked off in the view agenda. The alert message on top of the real-time window was triggered by an attempt to terminate the examination, and is a simple illustration of how cognitive aids can be integrated with image acquisition.

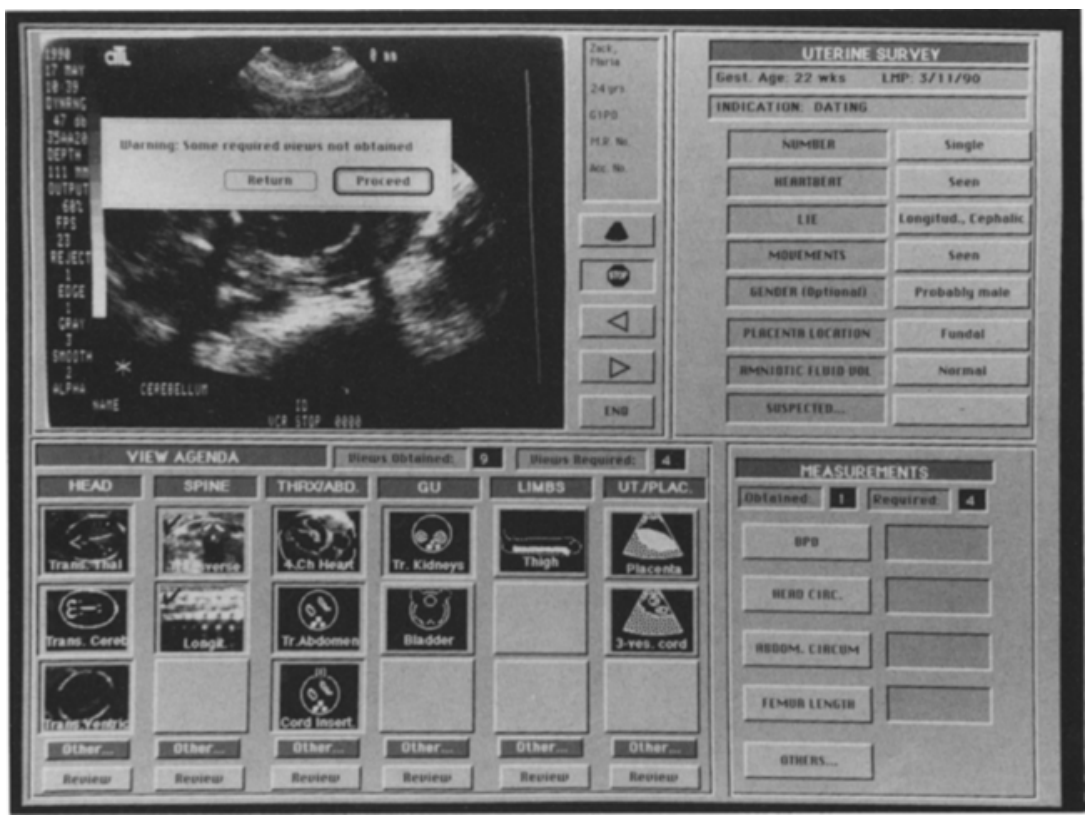

complete examination. They are designed to dynamically respond to typed findings and measurements so that an aberrant finding would prompt the sonographer to obtain more views in order to explore the finding and its possible causes and ramifications in greater detail. The presence of a hydronephrotic kidney, for example, would prompt the technologist to obtain further detailed views of the kidney and the rest of the urinary system in order to assess the extent of the hydronephrosis and discover the level of obstruction. This, of course, is exactly what an experienced technologist does automatically. In addition to providing this kind of benefit even to inexperienced operators, the system enforces thoroughness by ensuring that the technologist will be warned if the examination is terminated without obtaining all the prescribed views or measurements (Fig 1).

When a technologist obtains a particular view, he or she clicks on the appropriate icon. This freezes the real-time image and immediately brings up a new screen (Fig 2) that has (1) a checklist of all the things to look for in that particular view; (2) a list of points to check whether the view is along the right axis; (3) a date-specific reference image of that view with the appropriate landmarks and measurable structures labeled (these labels can be dragged with the mouse to the appropriate places to annotate the patient's frozen image, and the appropriate measurements can be similarly carried out on the frozen image); and (4) a group of smaller reference images showing acceptable ranges of pertinent normal variations and various pathologies encountered in that particular anatomic region at the given gestational age, in addition to acceptable and unacceptable offaxis views. If the technologist performs a measurement that is found to be abnormal for gestational age, the system gives a warning beep and goes further into pathologies or methodological errors that might have been responsible for the errant measurement (Fig 3): the cognitive feedback is immediate. If the image is acceptable it is recorded and the technologist is returned to the original screen, where that particular view is now checked off.

As the sonographer performs the examination, the system records and collates findings, creating a preliminary draft of the case report for use of the physician during reporting. A separate module also generates text and imagebased cognitive support for the physician during the process of case reporting.

Such a system is possible in obstetric ultrasound because some image interpretation is performed during image acquisition itself. The same strategy may be more difficult to apply in other domains. 


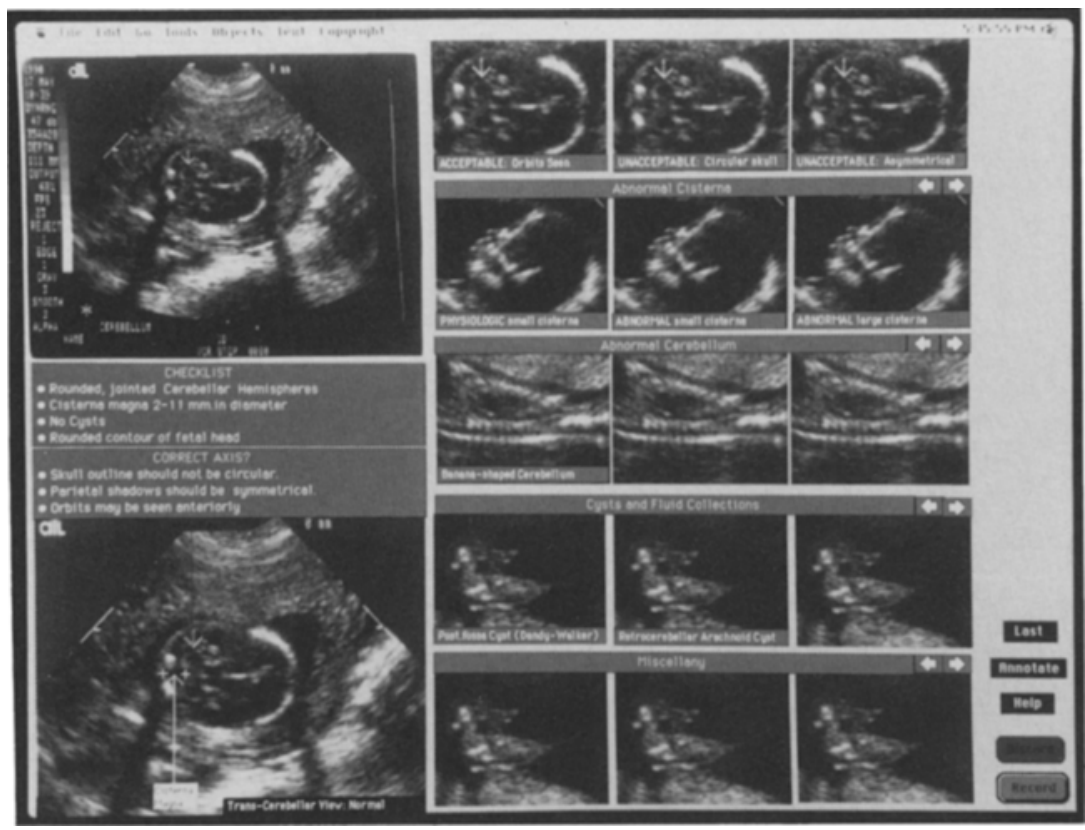

Fig 2. Detailed screen for the trans-cerebellar view showing frozen image, check-list of things to look for in current view, guidelines for assessing correctness of view, labeled reference image, and atlas of small images showing normal variants and pathologies encountered in given anatomical region and acceptable and unacceptable positioning of current view. (The actual small images used are placeholders and do not match their labels).

\section{Case Input During Case Reporting}

Computerized case reporting allows the combination of an expert system with a computerbased reporting system. This would be particularly suitable in those areas of radiology where there are a high proportion of normal examinations such as screening mammography where check-off forms are often used. Computer screens can be designed to mimic paper forms and can even improve substantially on them. IMAGE/ICON uses Apple Macintosh style dialog boxes and menus (Apple Computer Inc, Cupertino, CA) for entry of cases. We have also developed a screen-oriented form of data entry that attempts to minimize semantic variability by presenting the radiologist with visual choices rather than word choices. This system presents the radiologist with palettes of actual digitized

Fig 3. Warning message after the operator carried out a measurement of the cisterna magna in the trans-cerebellar view.

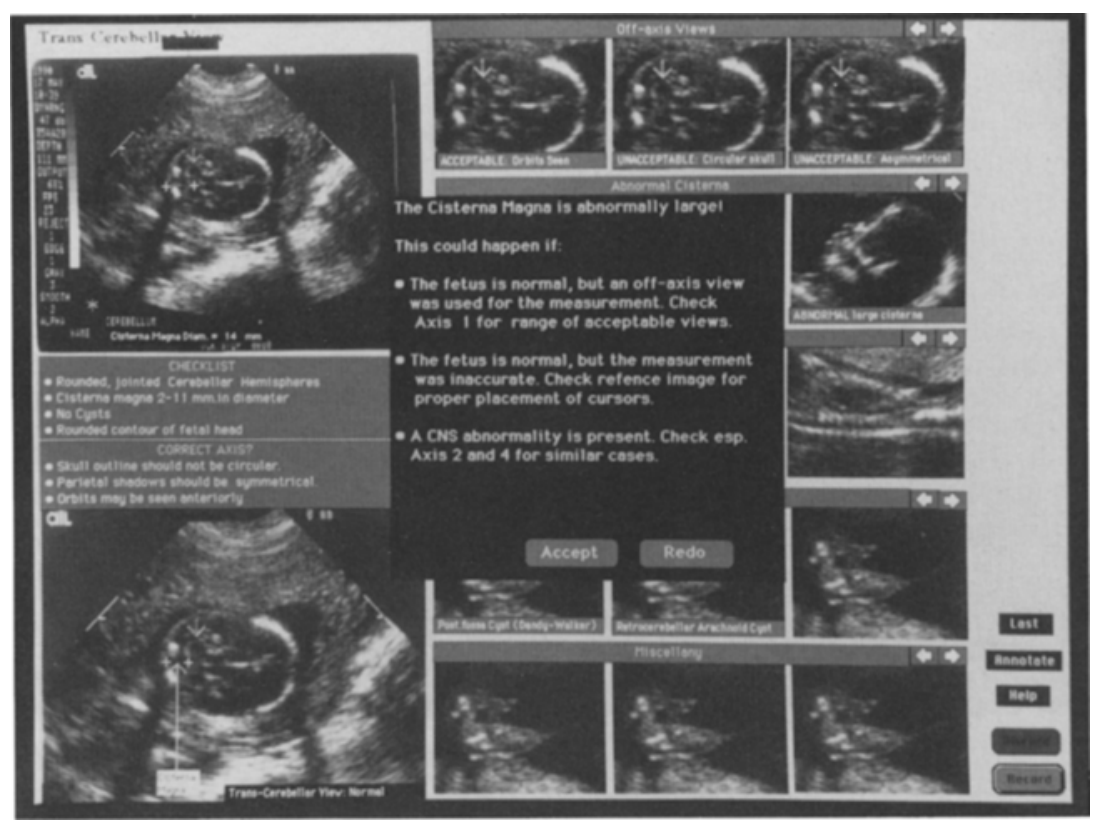


image features that may be selected to encode the findings present in a particular case (Fig 4).

In most areas of radiology, however, voice reporting is used almost exclusively. We have interfaced IMAGE/ICON to a commercial radiology voice reporting system (Voice RAD, Kurzweil Applied Intelligence Inc, Waltham, MA) that uses a trigger-phrase lexicon to generate radiographic reports. The resulting system, MAMMO/ICON, functions as a voice-activated expert system in the domain of mammography. ${ }^{13}$ Its input consists of exactly the same trigger words and phrases that a radiologist would speak into an automated film dictation system in order to produce a mammography report. Thus, if the radiologist says, "Mass small high density location subcutaneous," the voice system will enter the following sentences into the report: "There is a small high-density mass. The location of the mass is subcutaneous." At the same time, the case data is piped into the MAMMO/ICON system in the form of nameattribute-value tuples such as (mass present yes), (mass size small), (mass location subcutaneous), and so on. After the radiologist has finished dictating the report, which includes the tentative diagnosis, he or she can access the expert system's advice by saying, "Analyze." At this signal, the expert system evaluates the case tuples, generates a text critique, and retrieves a set of images relevant to the case (Fig 5). At this point, the radiologist can examine the critique and the images in detail, after which he or she has the option of changing the diagnosis before the final report is printed. If the case that the radiologist is viewing is routine, he or she may simply ignore IMAGE/ICON's output, but if there is anything unusual about the case, the system's advice is available to the radiologist with no extra effort.

Whereas this presents an ideal paradigm of expert system use, voice reporting using trigger phrases has many drawbacks and is unlikely to replace conventional voice dictation. For computerized voice dictation to be widely accepted, voice recognition technology must mature to the point where continuous speech recognition becomes possible. The integration of continuous speech with expert system advice would require sophisticated natural language parsing in order to translate the report into the internal symbolic form required by most expert systems. This is likely to be an extremely fruitful area for future research.

\section{DEFICIENCIES IN EXPERT SYSTEM CONTENT}

We can isolate the obstacles to expert system acceptance related to expert system content by assuming for a moment that the man-machine interface issues are completely solved. If there
Fig 4. Interface that presents visual choices for case description. A major finding type is selected by clicking on one of the four images in the top row (calcifications in this instance). This brings up a palette of icons (left) that allows the user to select the calcification subtype. Selection of "coarse calcifications" brings up a palette of images (center) from which the user can select the one most similar to the current case.

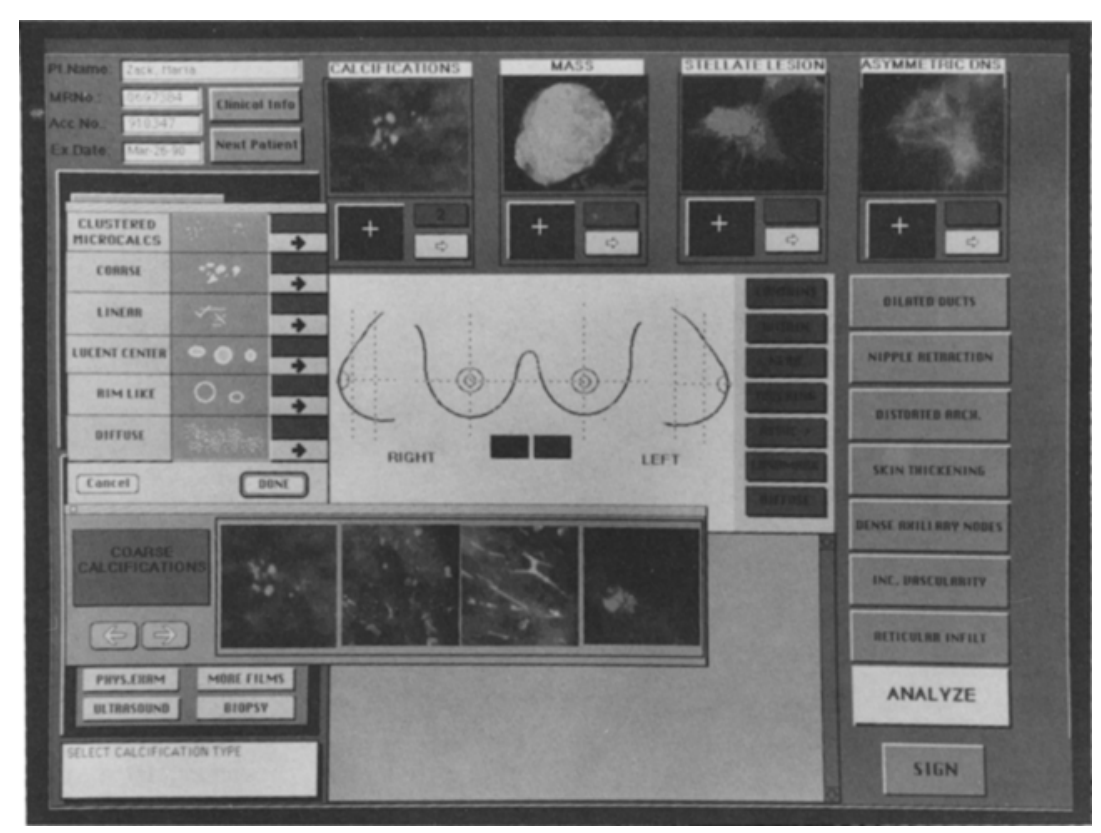




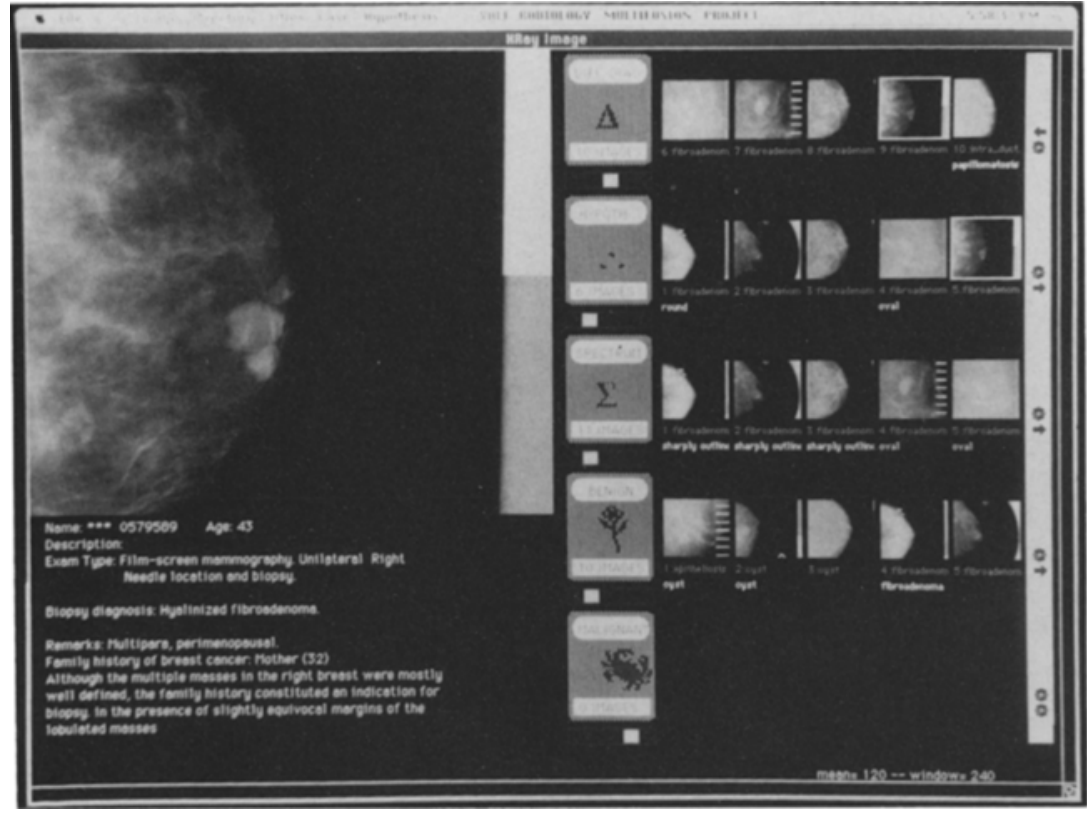

Fig 5. MAMMO/ICON image display. The large image on the left was retrieved by the system in response to the dictation of a case having multiple retroareolar masses. Each of the horizontal rows of images represents image axes: groups of images relevant to the dictated case in different ways. were an expert system that a radiologist could talk to, what kind of features would it need in order to be completely acceptable and useful? The guiding metaphor for our critiquing systems has been that of a friendly colleague, blessed with a perfect memory, who unobtrusively sits by the side of the radiologist as he or she reads a film. This expert can be turned to for a quick consultation in moments of uncertainty, and may occasionally draw one's attention to possible errors.

The question is whether we can accomplish this without having to provide so much world knowledge to the system that the task becomes the hopelessly more difficult one of creating an artificial human brain. Can the relevant expertise of a human radiologist be isolated from background world knowledge and still be useful? An instructive analogy has been made of an expert system to a movie set of, say, a building. It looks like the real object, but a small change in perspective will show that it lacks depth. What can be done to overcome this limitation without taking recourse to actually creating a real building? A careful movie-maker would make sure that his set possesses verisimilitude in all the possible views it is depicted within the movie. This is exactly what expert systems in medicine, and specifically in radiology, must incorporate in order to be useful. They must be able to help in some way with all the different kinds of decisions that the physician needs to make in a particular case.

In this regard, there are two major drawbacks of traditional medical expert systems: first, they tend to concentrate on single, distinct issues such as diagnosis and do not address all the decisions that the physician must make that are relevant to clinical outcome; and second, the kind of decision support required at different levels of training can vary, and the expert system must be able to satisfy the needs of different users or even the same user at different times.

\section{Clinical Outcome-Relevant Decision Support}

Many medical expert systems deal with clinically distinct areas such as diagnosis or workup. In most domains, however, the clinical outcome depends on the interplay of workup and diagnostic decisions. For instance, we have observed that in the domain of mammography, it is often far more difficult and clinically relevant to decide whether a small masslike lesion seen in only a single view is a real lesion or not than it is to sub-classify a benign lesion. In such a situation an expert typically recommends specific compression views that will best discriminate the lesion from superimposed parenchymal shadows. In a similar way, it is more important to know that a given finding requires an appro- 
priate management decision (biopsy or follow-up films) than it is to know a specific histologic diagnosis. It is important that such decision-making skills be a part of the expert system in addition to conventional diagnostic rules. To do so requires the construction of a robust computer model of the domain that incorporates notions of what issues are important.

Our original IMAGE/ICON systems dealt only with diagnostic issues. At present we are analyzing the different types of decisions that are made by mammographers during filmreading and how they influence clinical outcome.

\section{Training Level-Appropriate Decision Support}

In any domain, the kinds of issues that a person reading films struggles with vary a great deal, depending on the level of training. In mammography, for example, a person possessing some textbook knowledge of the domain may be able to recognize obvious lesions but may have trouble deciding whether a lesion is present or not in some cases. It generally takes some experience in order to calibrate one's understanding of what constitutes a normal variant and what constitutes a subtle lesion. At such a stage, presentation of prototypical images distinguishing between these two entities would be useful. At a later stage of training, however, when these differences have become obvious, presentation of such images may be obtrusive. Some training is needed in order to know which region of a particular breast view to examine to find a mass that is seen in a specific location in another view. It would certainly facilitate training if the expert system could highlight the area corresponding to the location of a lesion in another view. Once this is learned, however, the value of such an exercise may be questionable. Similarly, it is often the case that at an intermediate level of training a person may possess adequate skill at each individual step required in reading a mammogram (eg, identifying subtle masses and asymmetric densities, checking for calcifications, examining the nipple and skin, etc) but may not consistently go through all these steps in each and every case routinely. At this stage, having the expert system structure the reading session to ensure that the user goes through all the steps may help increase the user's competence. To a more advanced user, such a protocol may be second nature, and the expert system may be considered a burden rather than a help. At this stage the expert system must be more unobtrusive and must only give warnings when obvious oversights occur.

We obtained similar results in our informal evaluations of our ultrasound system prototype, which we demonstrated to 10 radiologists, obstetricians, and ultrasound technologists. It was unanimously felt that the system would be a useful teaching tool in the initial stages of a technologist's training. However, most of our respondents also felt that the system would not be of great value to more experienced sonographers and expressed the fear that it might slow them down.

In order to solve these problems, it is not only necessary for the system to have an internal model of the domain, but also a model of the types of skills that are needed to be an expert in the domain and some idea of the order in which these are acquired. Only then would the expert system be able to provide decision support in a way that is training level appropriate. It is obvious that these skills vary from domain to domain within the specialty of radiology. The identification of these skills and the delineation of their learning curves provides a challenge to knowledge engineers and system designers. A possible starting point for such efforts are the studies by cognitive scientists on the ways by which radiologists acquire expertise ${ }^{18,19}$ A project that uses these principles, with special emphasis on radiology education, is currently underway at Harvard Medical School and Bolt Beranek and Newman Inc. ${ }^{20}$

\section{Other Factors in Expert System Acceptance}

There are many other factors that can decrease expert system level acceptance, such as lack or inadequacy of explanation, obtrusiveness, too much or too little detail, too high or too low a level of knowledge, repetitiveness, and lack of accuracy and comprehensiveness of the knowledge. For the most part, most of them are related to the factors already mentioned. Thus, obtrusiveness and adequate detail and level of knowledge are related to calibration of the 
system to make it training level appropriate. Explanation is an important issue that has been successfully addressed in recent expert systems. In our systems, the text output is delivered in the form of a prose critique, which itself is a form of explanation. This is inherently more acceptable than a mere ranked list of differential diagnoses, because every statement made by the expert system is fully explained. The inclusion of a case summary for every image presented performs the same function with respect to the image output. Repetitiveness can be avoided to some extent by having a large image database and randomly replacing images with similar ones in different presentations of the same image group. Accuracy and comprehensiveness of the knowledge depends on how much knowledge engineering was done and how painstakingly it was performed. In limited domains such as mammography, a comprehensive system seems possible to attain by a small group working for 3 to 5 years. For larger domains, a more substantial effort may be required.

\section{CONCLUSION}

We have listed and described some of the obstacles that must be overcome before intelligent decision-support systems can be made available to radiologists in the clinical environment. Only continued research and development combined with continuous informal and formal clinical evaluation can help further define and overcome these hurdles. We are currently planning further development and formal evaluation studies of the MAMMO/ICON system with these aims in mind.

\section{REFERENCES}

1. Lodwick GS, Haun CL, Smith WE, et al: Computer diagnosis of primary bone tumors: A preliminary report. Radiology 80:273-275, 1963

2. Kahn CE, Messersmith RN, Jokich MD: PHOENIX-An expert system for selecting diagnostic imaging procedures. Invest Radiol 22:978-980, 1987

3. Swett HA, Rothschild M, Weltin GG, et al: Optimizing radiologic workup: An artificial intelligence approach. J Digital Imaging 2:15-20, 1989

4. Swett HA, Miller PL: ICON: A computer-based approach to differential diagnosis in radiology. Radiology 163:555-558, 1987

5. Swett HA, Fisher PR, Cohn AI, et al: Expert system controlled image display. Radiology 172:487-493, 1989

6. Piraino DW, Richmond BJ, Uetani M, et al: Problems in applying expert system technology to radiographic image interpretation. J Digit Imag 2:21-26, 1989

7. Cook HM, Fox MD: Application of expert systems to mammographic image analysis. Am J Physiol Imag 4:16-22, 1989

8. Ackerman LV, Burks MW, Rada R: Knowledge representation of CT scans of the head. Proc SPIE 454:443-448, 1984

9. du Boulay GH, Teather D, Morton BA, et al: Brain-a computer advisor system to aid in CT scan interpretation and cerebral disease diagnosis. Neuroradiology 29:196-199, 1987

10. Gross GW, Boone JM, Greco-Hunt V, et al: Neural networks in radiologic diagnosis. II. Interpretation of neonatal chest radiographic. Invest Radiol 25:1017-1023, 1990

11. Asad N, Doi $K$, MacMahon $H$, et al: Potential usefulness of an artificial neural network for differential diagnosis of interstitial lung disease: Pilot study. Radiology $177: 857-860,1990$
12. Piraino DW, Amartur SC, Richmond BJ, et al: Application of an artificial neural network in radiographic diagnosis. J Digit Imag 3:226-232, 1991

13. Fisher PR, Mutalik PG, Maddison I, et al: MAMMO/ ICON: Voice activated intelligent radiologic image display, in Arenson RL, Friedenberg RM (eds): SCAR-90Computer applications to assist radiology. Anaheim, CA, Symposia Foundation, 1990, pp 713-719

14. Giger ML, Yin FF, Doi K, et al: Investigation of methods for the computerized detection and analysis of mammographic masses. Proc SPIE 1233:183-184, 1990

15. Chan HP, Doi K, Galhotra S, et al: Image feature analysis and computer-aided diagnosis in digital radiography. 1. Automated detection of microcalcifications in mammography. Med Phys 14:538-548, 1987

16. Giger ML, Doi K, MacMahon H: Image feature analysis and computer-aided diagnosis in digital radiography. 3. Automated detection of nodules in peripheral lung fields. Med Phys 15:158-166, 1988

17. Katsuragawa S, Doi K, MacMahon H: Image feature analysis and computer-aided diagnosis in digital radiography: Classification of normal and abnormal lungs with interstitial disease in chest images. Med Phys 16:38-44, 1989

18. Lesgold AM, Feltovich PJ, Glaser R, et al: The acquisition of perceptual diagnostic skill in radiology. Learning Research and Development Center Technical Report No. PDS-1, Pittsburgh, PA, 1981

19. Myles-Worsley M, Johnston WA, Simons MA: The influence of expertise on X-ray image processing. J Exp Psychol 14:553-557, 1988

20. Rubin A: Contributions of cognitive science and educational technology to training in radiology. Invest Radiol 24:729-732, 1989 